\title{
THE RADIOLOGY OF CHRONIC PYELONEPHRITIS
}

\author{
C. J. Hodson, F.R.C.P., F.F.R. \\ $X$-ray Diagnostic Dept., \\ University College Hospital, W.C.1.
}

Chronic pyelonephritis is a generic term meaning different things to different people. To perhaps the majority it signifies the small, coarsely scarred kidney of what is also known as "atrophic pyelonephritis", which gives rise to hypertension in young people, or, if it is bilateral to renal failure and death. To some it embodies a histological picture which may be found in many conditions and which it is not always possible to associate closely with infection. To the clinician it means recurrent or relapsing urinary infection, and it is notoriously lacking in clearly defined clinical features in many instances. To the bacteriologist it is a state in which abnormal numbers of bacteria appear, or may be caused to appear, in the urine from time to time.

There is in fact no single entity which includes all these requirements, all the time. Nor indeed were it reasonable to expect one in so complicated an organ, any more than the term 'chronic lung infection' might be expected to refer to suppurative pneumonitis rather than cavitating tuberculosis.

Radiology's contribution to this subject is that it can define a coarse renal scar by excretion pyelography, and it enables both an early diagnosis to be made and the natural history of the disease to be studied.

The sign of a scar is a localised loss in thickness of the renal substance. There is usually disappearance of the adjacent pyramid also. (Fig. 1). The definition of this lesion, therefore, depends on the demonstration, during pyelography, of both the renal outlines and the calycine details, which in turn depends on care with radiographic technique and the preparation of the patient's abdomen before examination. We have shown that this is a perfectly possible everyday matter in a busy X-ray department. (Fig. 2).

Such scarring is essentially irregular in its distribution, albeit it tends to occur in certain patterns. It is therefore to be distinguished from the uniform change seen equally through-

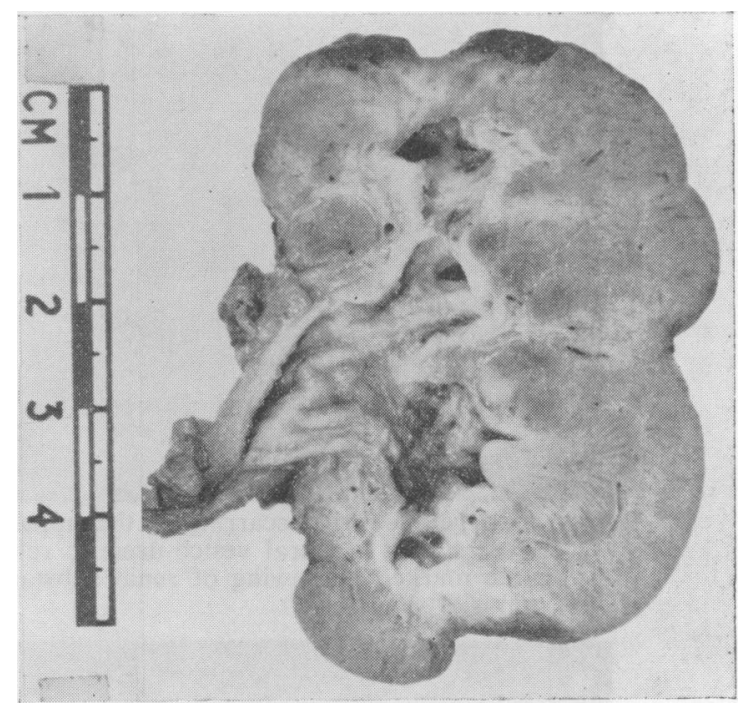

FIG. 1.-Pyelonephritic scarring. Female, 19 years. BP $190 / 120 \mathrm{~mm}$. Hg. No symptoms. Unilateral vesico-ureteric reflux. Specimen shows a normal pyramid and renal substance below which is a scar with absent pyramid and surface depression. (By kind permission of Messrs. J. \& A. Churchill Ltd., London).

out the kidney in back pressure atrophy, which also leads to gradual disappearance of renal pyramids and narrowing of renal substance. (Fig. 3).

It is to be distinguished from infarction in that in the latter condition the renal pyramid survives, although usually diminished in size, and the typical calyceal clubbing does not occur, while the distribution of infarcts also tends to be quite different from pyelonephritic scars, (Smith, 1962).

Reflux of urine from the bladder into the ureters, at rest or during micturition, is now an accepted concomitant of this gross form of scarring in a majority of cases, and can be demonstrated by the voiding cystourethrogram. (Fig. 4).

The scarred kidney, unless complicated by 


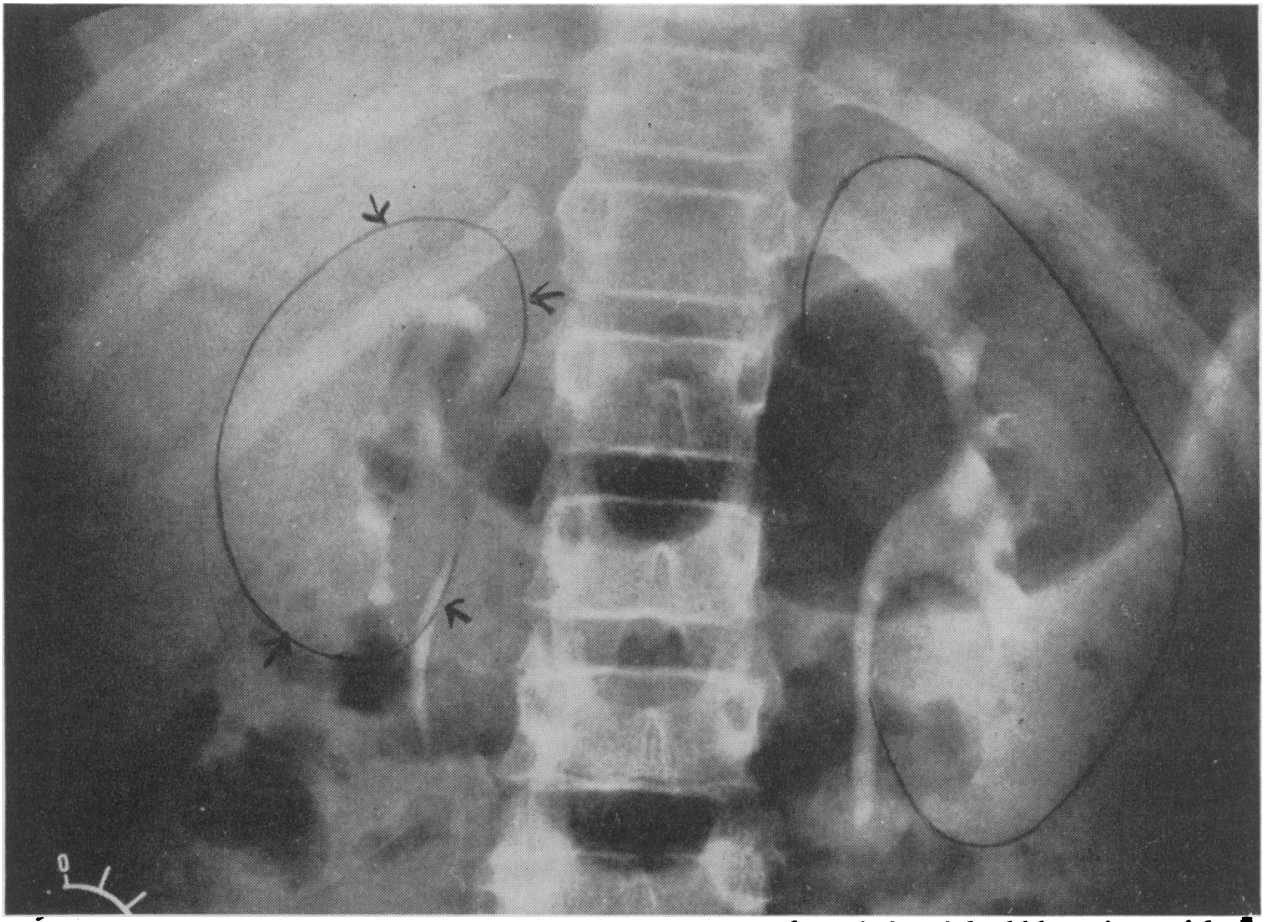

FIG. 2.-Pyelonephritic scarring in the upper and lower poles of the right kidney in a girl of 7 years with bilateral vesico-ureteric reflux. 2-year history of recurrent urinary infection. Note marked narrowing of renal substance compared with the rest of the kidney and the opposite side.

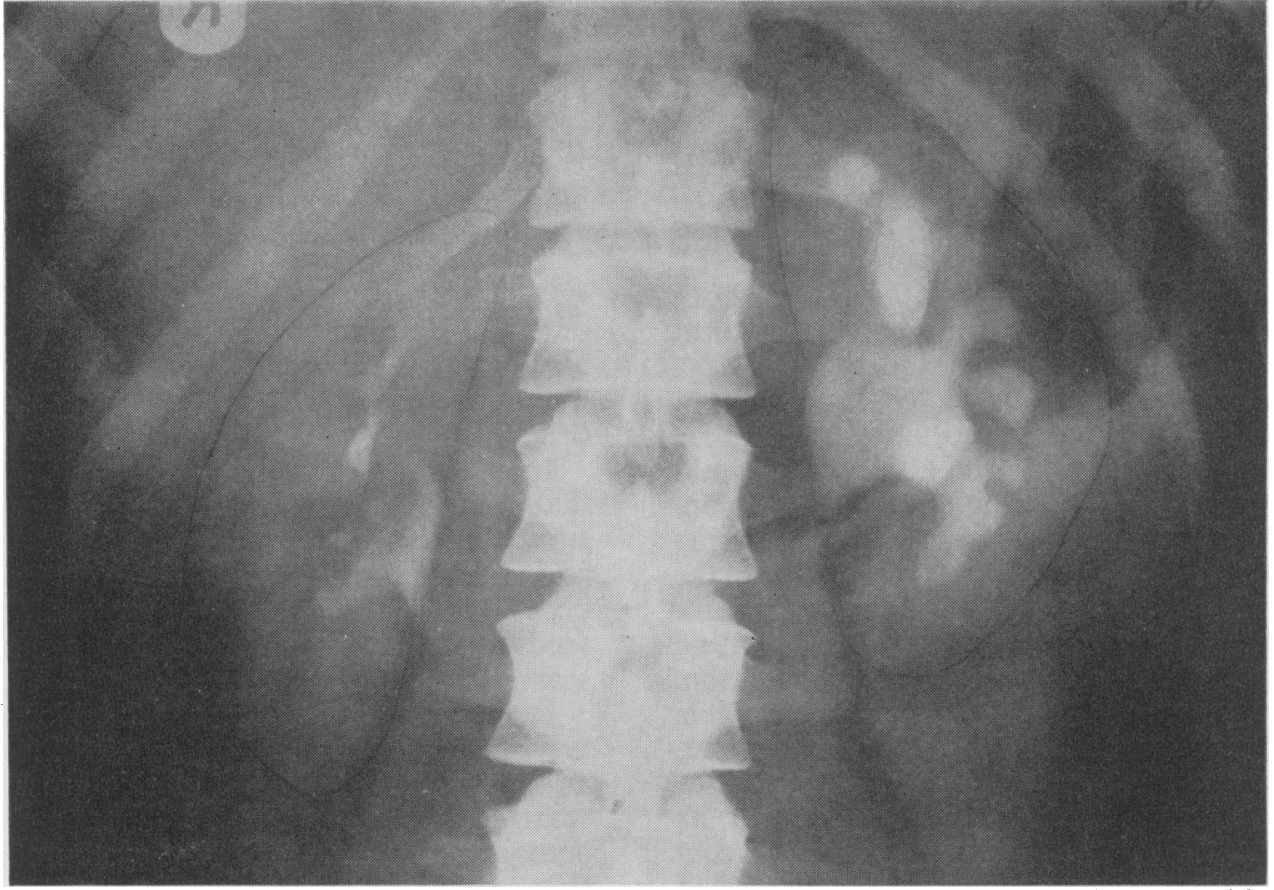

FIG. 3.-Back-pressure atrophy: IVP showing diffuse atrophy of the renal substance with uniform generalised blunting of the renal pyramids. Non-opaque stone in lowermost calyx. No reflux. 


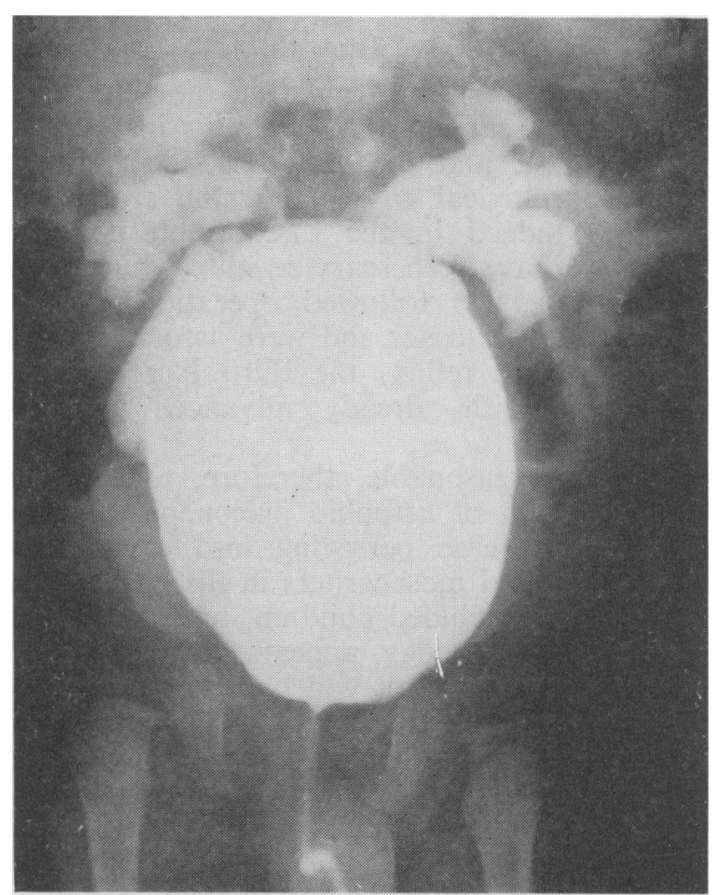

FIG. 4.-Severe bilateral vesico-ureteric reflux in a boy aged 20 months with an apparently normal urethra. Cystogram, before micturition has started, showing reflux at rest. Pyelonephritic scarring was present throughout both kidneys. some other factor such as previous hypertrophy, is reduced in size and progressive scarring can be seen by means of serial pyelograms to produce progressive shrinkage of renal tissue. (Fig. 5). In the growing kidney, moreover, a cessation of growth commonly follows, in unilateral cases, on the affected side; when bilateral, both kidneys tend to be small, one being more so than the other.

Having commenced the study of chronic pyelonephritis among adult patents referred largely from a hypertensive clinic, it soon became noticeable that there was a high incidence of such cases among children with urinary infection, and yet further experience revealed that coarse pyelonephritic scarring was the commonest renal lesion in the childhood population which is referred to hospital for investigation.

An analysis of our first 100 cases of renal scarring which were diagnosed radiologically confirmed this impression, the highest incidence of scarring being between 10 and 15 years. (Fig. 6). This in turn led to a comprehensive analysis of 200 consecutive cases of urinary infection in children under the age of 12 years, which has recently been published. (Smellie, Hodson, Edwards and Normand, 1964). This showed an incidence of pyelonephritic scars in $13 \%$ of patients investigated, all of them being under the age of 6 years.

The second impressive finding in these surveys is that the number of cases of scarring

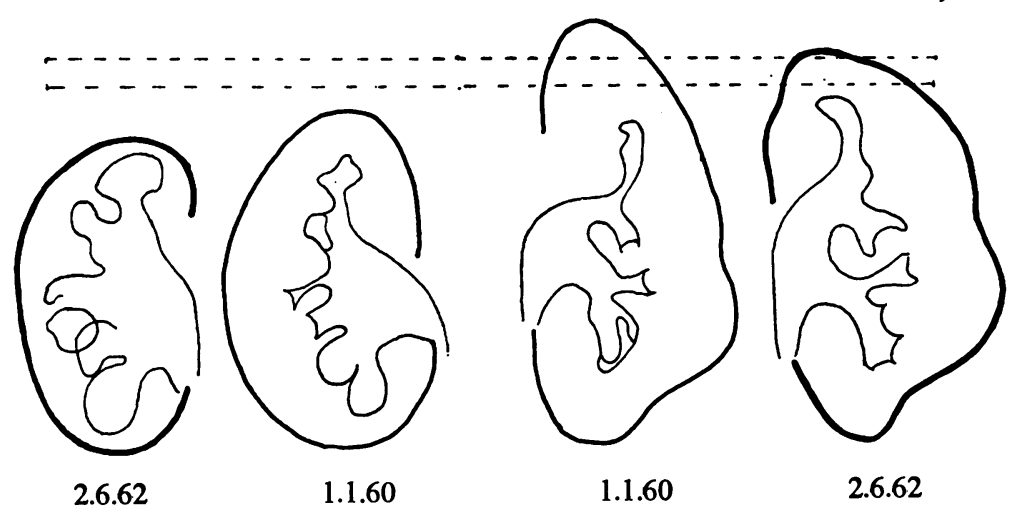

FIG. 5.-Progressive pyelonephritic scarring: tracings of the pyelograms of a girl at the ages of $4 \frac{3}{4}$ and 7 years. Persistent urinary infection and severe bilateral vesico-ureteric reflux was present. (The dotted lines represent the average kidney lengths for these ages.) 


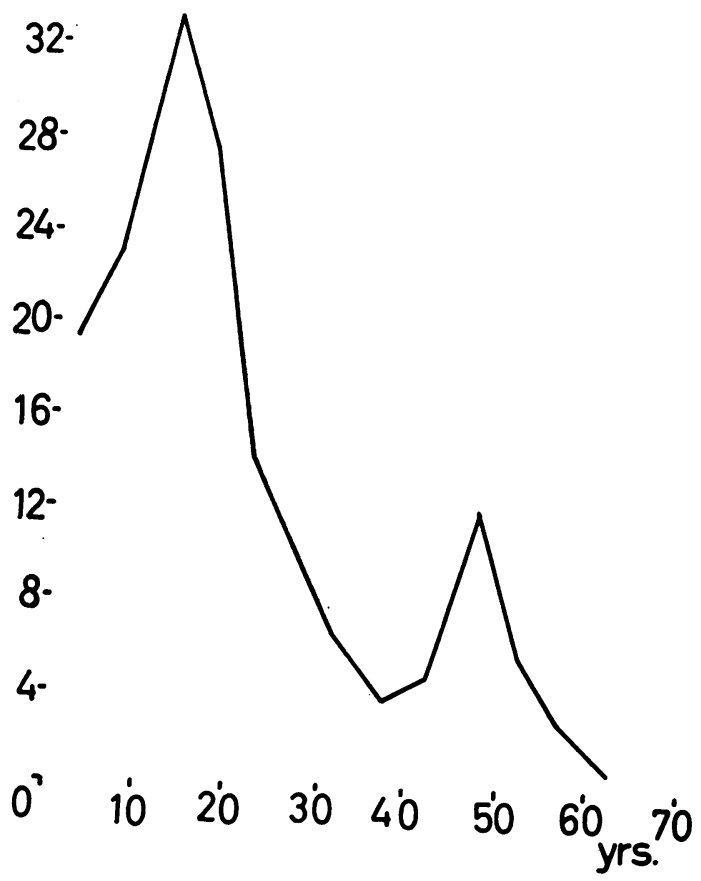

FiG. 6.-Graph showing the incidence of pyelonephritic scarring in 100 consecutive cases related to age (corrected for age incidence of pyelography examinations).

rapidly decreases up to the age of 45 years, with a small rise again between the ages of 50 and 65 years. This is particularly true of bilateral cases, and the only conclusion to be reached is that these patients die within the first three decades of life. In support of this is the large number of children showing rapid, $\stackrel{c}{c}$ progressive scarring when urinary infection is $\Rightarrow$ uncontrolled, and the very few adults (apart $\stackrel{5}{\rightarrow}$ from neurological cases) in which scarring is noticed. Indeed in the three adult cases in which we have seen scars develop while under observation, two followed operations on the lower end of a ureter and were associated with on vesico-ureteric reflux, the third happening in a patient with already advanced bilateral disease.

It seems reasonable, therefore, to look on this condition of atrophic pyelonephritis as a childhood disease persisting into early adult life, and indeed most writers in the past, whose studies have included children, have supported this view. Radiology appears to be a most valuable weapon with which to both diagnose and follow up these cases, and the problem as now defined is to evolve a simple and effective method of detecting urinary infection in the very young. Clinical methods are notoriously inadequate in this respect. What is required is a simple bacteriological technique applicable to out-patient and home surround ings.

\section{REFERENCES}

Smellie, J., Hodson, C. J., Edwards, D. and NORMAND, C. (1964): Clinical and Radiological Features of Urinary Infection in Childhood, Brit. med. J., ii, 1222.

SMITH, J. F. (1962): The Diagnosis of the Scars of Chronic Pyelonephritis, J. clin. Path., 15, 522. 